\title{
The influence of transcutaneous electrical neurostimulation (TENS) on human cerebral blood flow velocities
}

\author{
Mark ter Laan - J. Marc C. van Dijk • \\ Jan-Willem J. Elting $\cdot$ Vaclav Fidler $\cdot$ Michiel J. Staal
}

Received: 19 April 2010 / Accepted: 28 April 2010/Published online: 15 May 2010

(C) The Author(s) 2010. This article is published with open access at Springerlink.com

\begin{abstract}
Background It has been shown that transcutaneous electrical neurostimulation (TENS) reduces sympathetic tone. Spinal cord stimulation (SCS) has proven qualities to improve coronary, peripheral, and cerebral blood circulation. Therefore, we postulate that TENS and SCS affect the autonomic nervous system in analogous ways. In this line of thought, cervical application of TENS might be a useful and simple adjunct in the treatment of cerebrovascular disease by improving cerebral blood flow. Experiments were performed in order to assess whether cervical TENS is safe and whether an effect on cerebral blood flow velocity (CBFV) can be shown in healthy subjects.

Method A controlled, non-randomized, phase 1 study was performed with 20 healthy volunteers. Cervical TENS was applied in several frequencies, with and without hyperventilation. Continuous registration of blood pressure, pulse, CBFV (estimated by transcranial Doppler sonography) and end-tidal carbon dioxide concentration was performed.

Findings Cervical TENS was well-tolerated by all subjects. Despite small effects on heart rate (HR) and mean arterial
\end{abstract}

M. ter Laan $(\bowtie) \cdot$ J. M. C. van Dijk $\cdot$ M. J. Staal Department of Neurosurgery, University Medical Center Groningen, University of Groningen,

P.O. Box 30.001, 9700 RB Groningen, The Netherlands

e-mail: m.ter.laan@nchir.umcg.nl

J.-W. J. Elting

Department of Neurology, University Medical Center Groningen,

University of Groningen,

Groningen, The Netherlands

V. Fidler

Department of Epidemiology, University Medical Center

Groningen, University of Groningen,

Groningen, The Netherlands blood pressure (MAP), a significant effect on middle cerebral artery (MCA) blood flow velocity was not demonstrated. No effect of age, gender, current or session order on MCA, HR, or MAP was found. TENS did not influence the effect of hyperventilation.

Conclusions In these experiments, application of cervical TENS is proven to be a safe procedure. However, no effects on cerebral blood flow velocity could be detected, perhaps due to the intact cerebral autoregulation in the healthy volunteers.

Keywords Cerebrovascular circulation - Transcutaneous electric neurostimulation - Intracranial vasospasm .

Sympathetic nervous system

\section{Introduction}

Spinal cord stimulation (SCS) is widely used in the treatment of pain. Although several mechanisms have been proposed, its exact mechanism of action remains unclear [13]. At least part of the effects of SCS is mediated by the autonomic nervous system, as shown by the fact that sympathetically mediated pain (e.g., visceral pain and complex regional pain syndromes) responds better than nociceptive pain to SCS treatment [13]. In addition, it has been demonstrated that SCS has autonomic (side)effects, such as increasing peripheral blood flow in Raynaud's phenomenon, peripheral ischemic pain, coronary disease, and complex regional pain syndromes $[4,11,13]$. Since the sympathetic fibers are located ventrally in the spinal cord and SCS electrodes are placed dorsally, these ventral fibers are not located within the electrical field. Therefore, these autonomic effect must be indirect, e.g., via antidromic pathways [13]. 
Transcutaneous electrical neurostimulation (TENS) has been used since 1974 to test the tolerance for stimuli in the work-up for SCS [1]. Some of the effects seen in SCS can be produced by TENS as well. Effects on peripheral blood flow have been shown, as well as increased coronary perfusion [12]. An experiment on autonomic cardiovascular reflexes demonstrated a decreased sympathetic response when using TENS [17]. Since the sympathetic effects as shown by SCS must be indirect effects of the electrical stimulation, we postulate that TENS produces these effects in the same way.

In several studies, an increase of cerebral blood flow (CBF) has been described as a result of cervical SCS [2, 3, $9,10]$. The effects of SCS on CBF are most likely mediated through the sympathetic nervous system, since cerebral vasculature is highly sympathetically innervated and the vascular diameter is related to sympathetic tone [18]. Patel showed that this effect is mediated via a central pathway [15]. This pathway involves brainstem vasomotor centers that connect with the cerebral microvasculature through intrinsic nerve pathways. In experiments on rats, he showed that surgical sympathectomy failed to influence the $\mathrm{CBF}$ response caused by cervical SCS, while transsection of the spinal cord at the cervicomedullary junction completely abolished all increases in CBF. Taking into account the proven effect of TENS on peripheral blood flow and its (indirect) effect on sympathetic tone, TENS should be able to alter CBF, an analog to SCS. As such, TENS could be a useful non-invasive adjunct in the treatment of cerebrovascular disease (CVD).

TENS either has a direct effect on vascular diameter or has an effect on the sympathetic tone. If the latter is true, then, in resting conditions, there should be no effect on CBF. Therefore, in order to find out whether the sympathetic tone is reduced by TENS, the effects of TENS on $\mathrm{CBF}$ in hyperventilating subjects is of interest. Given that cerebral blood flow in response to hyperventilation might partially be sympathetically mediated $[7,8]$, this response should be reduced by TENS.

As cervical TENS is a novel potential therapeutic application in the treatment of CVD, this paper describes the experiments that were performed to assess the effects of TENS on CBF in healthy subjects and to register its potential side effects.

\section{Materials and methods}

\section{Study design}

The study was designed as a controlled (subjects were their own controls), non-randomized phase 1 experiment. Healthy volunteers were recruited from the general public by advertisement in the local newspapers. Cerebral blood flow velocity $(\mathrm{CBFV})$ can be seen as a representation of $\mathrm{CBF}$ when the vascular cross-sectional area (CSA) remains constant $(\mathrm{CBFV}=\mathrm{CBF} / \mathrm{CSA})$. Cerebral blood flow velocity was measured by transcranial Doppler (TCD) of the medial cerebral artery (MCA). The trial has been authorized by the local Research Ethical Board and the Dutch Central Commission of Human Research.

\section{Sample size calculation}

The literature reports inter-individual standard deviation of flow velocities as about $15 \%$ [21] and the intra-individual standard deviation as $8 \%$ [14]. Sample size calculation is based on the requirement that a difference in flow velocities of $20 \mathrm{~cm} / \mathrm{s}$ (ca 20\%) between frequencies 2 and $120 \mathrm{~Hz}$ should be detected with a probability (power) of $90 \%$ when testing at a significance level of 5\% two-sided. This results in a sample size of 20 .

\section{Treatment of subjects}

The subjects were treated with conventional TENS, providing a continuous flow of symmetrical rectangular biphasic pulses (Schwa Medico, Pierenkemper GMBH; Wetzlarestr. 41-43, D-35630, Ehringhausen, Germany). Stimulation was performed at $90 \%$ of the highest tolerated current, with a pulse-width of $200 \mathrm{~ms}$. Stimulation frequencies were $2,20,50,100$, and $120 \mathrm{~Hz}$. In this way, the entire range of possible TENS frequencies and the most used frequencies in current TENS treatments were captured. Subjects were supine during the entire experiment. The TENS electrodes were applied cervical at the height of the mandible on both sides of the nuchal ligament (dermatome C2-C3). Doppler probes were applied to the skull for continuous registration of flow velocity in the MCA (left and right). Continuous registration of pulse and blood pressure was recorded by plethysmography.

For the first experiment (Fig. 1), five blocks of measurements took place for each TENS frequency, $5 \mathrm{~min}$ of registration without TENS, and followed by $5 \mathrm{~min}$ with TENS (so each TENS frequency had its own controlbaseline). The order of frequencies was randomized (using the QuickCalcs online calculator, GraphPad Software, Inc.) in order to correct for possible alteration in time of the sympathetic tone during the experiment.

The second experiment (Fig. 2) was designed to examine whether TENS influences the normal cerebral regulation that physiologically occurs in reaction to hyperventilation. In the same way as the first experiment, blocks of measurements were recorded, now at a fixed frequency of $100 \mathrm{~Hz}$. For practical purposes, only one frequency was used for this experiment. The frequency of $100 \mathrm{~Hz}$ was 


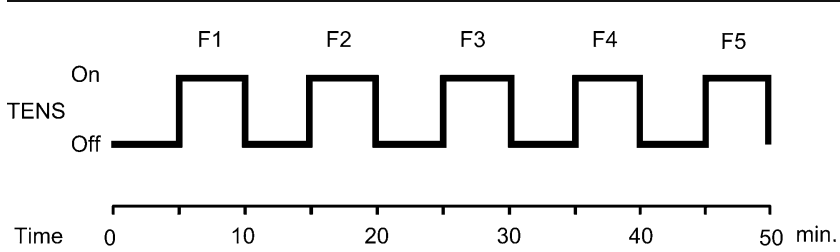

Fig. 1 Set-up of experiment 1: five blocks of baseline measurements followed by 5 -min measurement with TENS in randomized frequencies 1 to 5

chosen because it has been used in previous TENS experiments to alter blood flow in coronary arteries [5]. During this part of the experiment, end-tidal partial carbon dioxide concentration (ETCO2) was measured in the exhaled air as a measure of hyperventilation. Hyperventilation was defined as a decrease of ETCO2 to $2 \%$. Measurements were performed following the scheme below (in half of the subjects the order was reversed, i.e., first TENS with hyperventilation):

- Three minutes registration with hyperventilation (1 min to achieve an ETCO2 of 2\%, 2 min measurement)

- Ten minutes registration without TENS/hyperventilation

- Three minutes registration with TENS and hyperventilation (1 min to achieve an ETCO2 of 2\%, 2 min measuring)

- Ten minutes registration without TENS or until baseline variables were approached

\section{Data processing}

Data was collected using a continuous TCD monitor (DIGILITEtm, Raanana, Israel), a plethysmograph for assessing blood pressure and pulse (Finometer-Pro, Finapress Medical Systems, Amsterdam, The Netherlands), an electrocardiograph (Finometer-Pro, Finapress Medical Systems, Amsterdam, The Netherlands), and a capnograph (Capnomac Ultima, GE Healthcare, Chalfont St Giles, UK). All analog output was

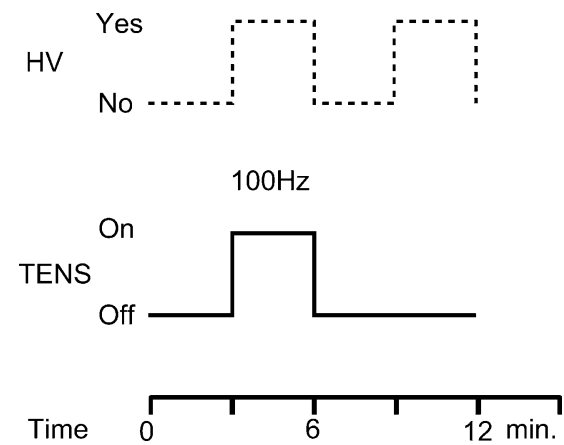

Fig. 2 Set-up of experiment 2: two blocks of baseline measurements, followed by a measurement with hyperventilation either with or without TENS. Order of measurements was reversed in half of subjects routed to a computer via a digitizer. The data were continuously registered using Neuromon (incorporating Labview 7.1, National Instruments) software. Before statistical analysis, all artifacts were deleted from the data streams (e.g., reduced Doppler signal due to transducer dislodgement). In order to clean the data from noise, filtering was performed using in-house written routines in the Matlab environment (The Math Works, Inc., Natwick, MA, USA, Matlab 6.5). Bandwidths for the data were defined (50-150 bpm for HR, $5-100 \mathrm{~cm} / \mathrm{s}$ for MCA, $60-180 \mathrm{mmHg}$ for ABP, and $2-6 \%$ for $\mathrm{CO} 2$ in resting phase), and top and bottom $10 \%$ of data were deleted, replacing those by linear interpolation. Next, 3-min data streams were taken from each measurement, in each case a stream from -4 to $-1 \mathrm{~min}$ before the start of TENS and a stream from +1 to +4 min after the start of TENS. From the hyperventilation data, a stream was taken from -2.5 to $-0.5 \mathrm{~min}$ from the start of $\mathrm{HV}$ and another 2-min stream as soon as ETCO2 had stabilized around 2-2.5\% (app. 15$20 \mathrm{mmHg}$ ). Average values of all streams were calculated and used for statistical analysis.

\section{Statistical analysis}

The purpose of the analysis was to find out (1) the effect of different TENS frequencies on cerebral blood flow and (2) whether the TENS frequency of $100 \mathrm{~Hz}$ modifies the effect of hyperventilation on cerebral blood flow. The data from the first experiment were used to address the first question and the data from the second experiment to address the second question. For each TENS frequency, we subtracted the measurement at the preceding non-TENS session from the measurement with TENS and used these "baselinecorrected" differences as basic outcomes in the analysis. We used all observations from the MCA side which had the highest blood flow velocity at baseline as measured by TCD. For both questions, the analysis consisted of fitting mixed effects regression models to the data [16]. For question 1, we have used the TENS frequency (at five levels), the order of observations, age, and gender of the participants as fixed effect explanatory variables. For question 2 , the fixed effects were TENS frequency ( 0 or 100), ETCO2, age, and gender. Statistical tests were carried out at $5 \%$ level of significance. We used the statistical package $\mathrm{R}$ for computations (version 2.8.1; library nlme).

\section{Results}

A group of 11 females and nine males, all healthy subjects, completed the experiment, in age ranging from 25 to 65 years (median 46.5 years). Two more volunteers participated, but their measurements failed. In one individual, the temporal bony windows were insufficient to obtain 
Fig. 3 Box plots of mean values of cerebral blood flow velocities in MCA (centimeters per second), heart rate (HR; beats per minute), and mean arterial pressure (MAP; millimeters of $\mathrm{Hg}$ ) at the different TENS frequencies (a $0=$ baseline). The plots in b show the differences of the mean values when compared with baseline
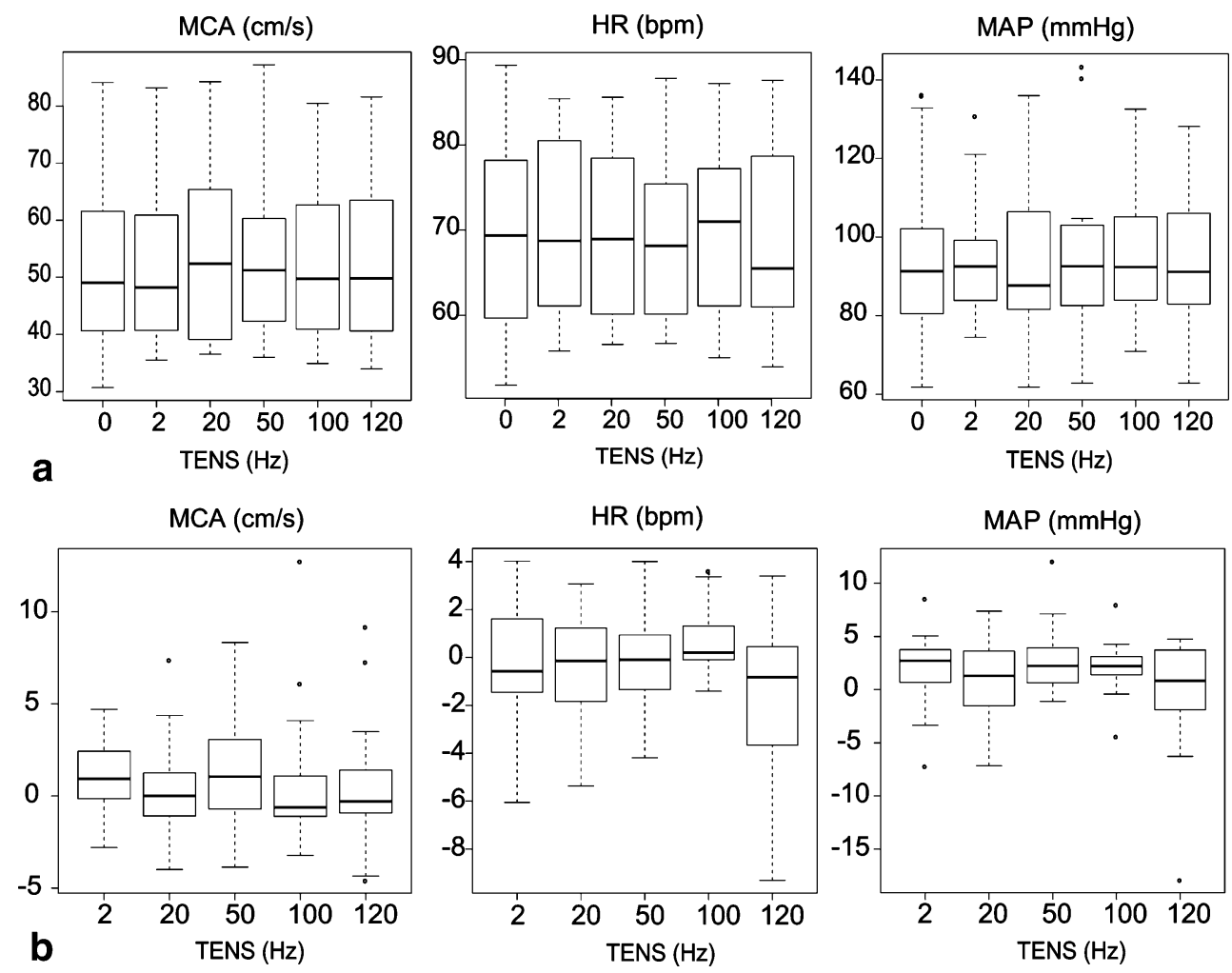

adequate MCA signals. In the other, an exceptional amount of noise was perceived on all signals; therefore, the experiment was aborted.

All volunteers tolerated TENS well, though the highest tolerated current did vary from 10 to $35 \mathrm{~mA}$. The only adverse events reported by the volunteers were pressure from the cap used to position the TCD probes and tingling sensations or dizziness as a result of the hyperventilation. All volunteers reached the predefined threshold of an ETCO 2 of $2 \%$ during hyperventilation.

Figure 3a shows box plots with values of MCA blood flow velocities, blood pressures (MAP), and pulse (HR) without TENS and per TENS frequency for the first experiment. Box plots in Fig. 3b show differences between
MCA blood flow velocities, MAPs and HR, and their coupled baseline values. The mean differences and the corresponding confidence intervals for MCA, HR, and MAP are shown in Table 1. The effect of TENS on MCA was on average $0.78 \mathrm{~cm} / \mathrm{s}(95 \% \mathrm{CI} ; 0.06$ to 1.50$)$, the effects at different TENS frequencies were not statistically significantly different $(P=0.80)$. For HR and MAP, too, the effects of different TENS frequencies did not differ $(P=$ 0.06 and $P=0.14$, respectively). Compared with the baseline, the HR was significantly lower at $120 \mathrm{~Hz}$, and the MAP was significantly higher at 2, 50, and $100 \mathrm{~Hz}$. However, the effects are so small, that they cannot be judged clinically relevant. No effect of age, gender, or session order on MCA, HR, or MAP was found.

Table 1 Differences of MCA blood flow velocities (MCA), heart rates (HR), and mean arterial blood pressures (MAP) for each TENS frequency compared with their coupled baseline values

\begin{tabular}{lllllll}
\hline Frequency & $\begin{array}{l}\text { MCA } \\
\text { Effect }\end{array}$ & $95 \%$ CI & $\begin{array}{l}\text { HR } \\
\text { Effect }\end{array}$ & $95 \%$ CI & \multicolumn{2}{c}{$\begin{array}{l}\text { MAP } \\
\text { Effect }\end{array}$} \\
\hline $2 \mathrm{~Hz}$ & 1.019 & $(-0.277,2.315)$ & -0.233 & $(-1.410,0.944)$ & $2.133^{\mathrm{a}}$ & $(0.720,3.547)$ \\
$20 \mathrm{~Hz}$ & 0.399 & $(-0.897,1.695)$ & -0.453 & $(-1.750,0.844)$ & 0.876 & $(-0.570,2.322)$ \\
$50 \mathrm{~Hz}$ & 1.247 & $(-0.049,2.543)$ & -0.116 & $(-1.369,1.137)$ & $2.565^{\mathrm{a}}$ & $(1.152,3.979)$ \\
$100 \mathrm{~Hz}$ & 0.874 & $(-0.423,2.170)$ & 0.713 & $(-0.500,1.927)$ & $2.180^{\mathrm{a}}$ & $(0.766,3.593)$ \\
$120 \mathrm{~Hz}$ & 0.347 & $(-0.931,1.661)$ & $-1.844^{\mathrm{a}}$ & $(-3.057,-0.631)$ & 0.668 & $(-0.778,2.115)$ \\
Overall & 0.781 & $(0.060,1.501)$ & -0.390 & $(-0.971,0.191)$ & 1.708 & $(0.825,2.591)$ \\
\hline
\end{tabular}

${ }^{a}$ Significant effect 
Fig. 4 Box plots of mean values of MCA blood flow velocities (centimeters per second), heart rate (HR; beats per minute), and mean arterial pressure (MAP; millimeters of $\mathrm{Hg}$ ), for baseline (cl and $c 2$ ), hyperventilation $(H V)$, and hyperventilation combined with TENS $(H V+; \mathbf{a})$ Differences of the mean values of TENS with (TENS+) and without (TENS-) hyperventilation, compared with their baselines are shown in (b)

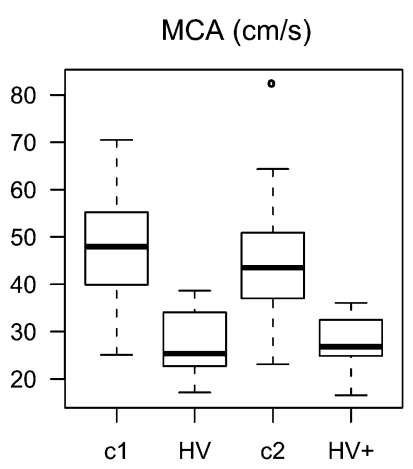

a

$\operatorname{MCA}(\mathrm{cm} / \mathrm{s})$

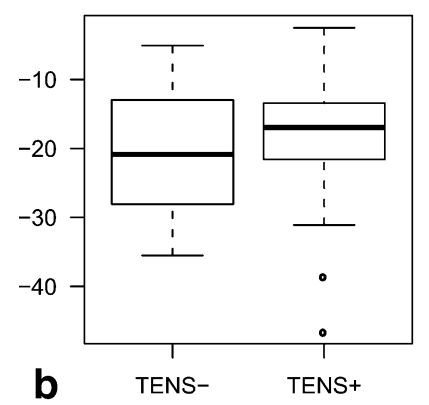

Figure $4 \mathrm{a}, \mathrm{b}$ show box plots of values of MCA blood flow, blood pressure, and pulse for experiment 2: with and without TENS, and with and without hyperventilation. No significant effect of TENS on MCA, HR, or MAP on the effect of hyperventilation was detected ( $P$ values $0.43,0.58$, 0.31 , respectively). ETC02 (difference between the measurements with and without hyperventilation) was found to be positively related to MCA and negatively to $\mathrm{HR}(P<0.001)$.

Because the highest tolerated current varied between volunteers from 10 to $35 \mathrm{~mA}$, we have also included the current in the fixed-effects model. No statistically significant effects of current could be found.

\section{Discussion}

We have studied whether TENS can influence the cerebral blood flow in healthy subjects. We postulated that TENS might influence cerebral blood flow via sympathetic pathways as observed in experiments with SCS [2, 3, 6, 9-11, $13,15]$. Also, TENS has been shown to increase locoregional blood flow and sympathetic tone $[12,17]$.

The purpose of this study was twofold: (1) to find out if the use of cervically applied TENS is safe and (2) to find out the effect on CBF in healthy subjects.

We suggested TENS might either (indirectly) create a sympathetic response that influences CBF or TENS might alter the sympathetic tone. To test the first way of action, we performed experiment 1 in which CBF was measured during stimulation with several TENS frequencies in resting
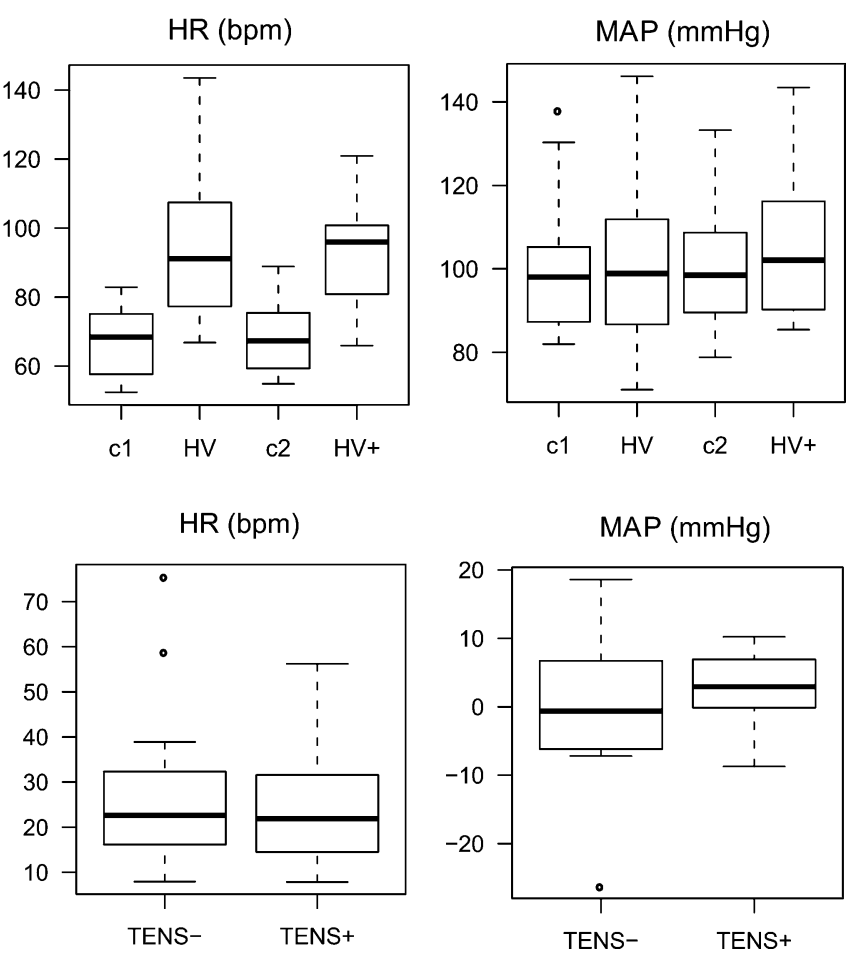

healthy subjects. If only sympathetic tone is influenced by TENS, subjects in rest will not show an effect, but subjects that have to apply their autonomous nervous system might. Because the cerebral response to carbon dioxide changes might be partially sympathetically mediated [7, 8], we have also tested whether TENS influences the response to hyperventilation.

Because sympathetic tone can easily change during an experiment (an individual can become either more or less comfortable during the experiment), we have applied several measures to compensate for this effect. Firstly, we have randomized the order of applied TENS frequencies; secondly we have provided each frequency with its own control-baseline-measurement, and thirdly, the order of TENS with/without hyperventilation varied.

TENS was tolerated very well by all subjects. No adverse events were found. Also, systemic blood pressure and heart rate were not affected by TENS in a clinically relevant manner.

As for the effect of TENS on CBF, no statistically significant effects were found for the individual TENS frequencies, neither at rest nor in the hyperventilation experiment. Overall, there was a small increase of MCA blood flow velocity $(0.78 \mathrm{~cm} / \mathrm{s})$ when TENS is compared with rest. The fall in MCA blood flow velocity as a result of hyperventilation was a little less prominent when TENS was applied, but this did not reach statistical significance.

Several explanations can be advanced as to why no effect was found. Firstly, of course, there might be no effect at all. Secondly, TENS might not be powerful enough 
because subjects can only tolerate a certain amount of current. However, we did not find a difference between volunteers that tolerated higher and volunteers that tolerated lower currents. Thirdly, we have only used one frequency in the hyperventilation experiment; possibly, other frequencies give different results. Fourthly, one might have to apply TENS for a longer period, although we do not think this is plausible, given the results of other TENS studies. Finally, regulatory mechanisms of $\mathrm{CBF}$ are complex. $\mathrm{CBF}$ depends on several parameters including MAP, ICP, CO2, $\mathrm{O} 2$, and other (auto)regulatory mechanisms. The role of the sympathetic nervous system on these parameters remains unclear. Sympathetic tone does regulate MAP by means of peripheral vasoconstriction in organ beds, but there is ongoing debate about the sympathetic effects on cerebral vasculature $[19,20]$. Also, the effects of $\mathrm{CO} 2$ might be far stronger than the sympathetic effects; possibly, sympathetic tone only has a modulating effect [8]. Moreover, the study was performed in healthy subjects with a powerful, intact autoregulation. This might have masked the effects of TENS on sympathetic tone.

The power analysis was aimed at finding a clinically significant effect; thus, with our experiments, we can only conclude that there seems to be no such effect in a healthy population. This does not yet prove that TENS does not influence the sympathetic nervous system and CBF, especially because we did notice a tendency towards less decreased blood flow velocities in reaction to hyperventilation when TENS was applied.

Possibly, an effect of TENS is appreciated in patients with disturbed autoregulation, e.g., patients with cerebral ischemia or following subarachnoid hemorrhage (with locally disturbed blood-brain barrier). Since cervical TENS did not have a clinically relevant effect on systemic blood pressure and heart rate, TENS can be safely applied to those patients. We think it is important to further investigate the effect of TENS on CBF in the presence of a disturbed autoregulation, especially since the influence of sympathetic tone is possibly much more significant in these pathological situations.

Open Access This article is distributed under the terms of the Creative Commons Attribution Noncommercial License which permits any noncommercial use, distribution, and reproduction in any medium, provided the original author(s) and source are credited.

\section{References}

1. Burton C (1974) Instrumentation for dorsal column stimulator implantation. Surg Neurol 2(1):39-40
2. Clavo B, Robaina F, Catala L, Perez JL, Lloret M, Carames MA, Morera J, Lopez L, Suarez G, Macias D, Rivero J, Hernandez MA (2004) Effect of cervical spinal cord stimulation on regional blood flow and oxygenation in advanced head and neck tumours. Ann Oncol 15(5):802-807

3. Clavo B, Robaina F, Catala L, Valcarcel B, Morera J, Carames MA, Ruiz-Egea E, Panero F, Lloret M, Hernandez MA (2003) Increased locoregional blood flow in brain tumors after cervical spinal cord stimulation. J Neurosurg 98(6):1263-1270

4. Cook AW, Oygar A, Baggenstos P, Pacheco S, Kleriga E (1976) Vascular disease of extremities. Electric stimulation of spinal cord and posterior roots. N Y State J Med 76(3):366-368

5. De Vries J, Anthonio RL, DeJongste MJ, Jessurun GA, Tan ES, de Smet BJ, van den Heuvel AF, Staal MJ, Zijlstra F (2007) The effect of electrical neurostimulation on collateral perfusion during acute coronary occlusion. BMC Cardiovasc Disord 7:18

6. Hautvast RW, Ter Horst GJ, DeJong BM, DeJongste MJ, Blanksma PK, Paans AM, Korf J (1997) Relative changes in regional cerebral blood flow during spinal cord stimulation in patients with refractory angina pectoris. Eur J Neurosci 9(6):1178-1183

7. Jordan J, Shannon JR, Black BK, Paranjape SY, Barwise J, Robertson D (1998) Raised cerebrovascular resistance in idiopathic orthostatic intolerance: evidence for sympathetic vasoconstriction. Hypertension 32(4):699-704

8. Jordan J, Shannon JR, Diedrich A, Black B, Costa F, Robertson D, Biaggioni I (2000) Interaction of carbon dioxide and sympathetic nervous system activity in the regulation of cerebral perfusion in humans. Hypertension 36(3):383-388

9. Karadag O (2005) Cervical spinal cord stimulation increases cerebral cortical blood flow in an experimental cerebral vasospasm model. Acta Neurochir (Wien ) 147(1):79-84

10. Lee JY, Huang DL, Keep R, Sagher O (2008) Effect of electrical stimulation of the cervical spinal cord on blood flow following subarachnoid hemorrhage. J Neurosurg 109(6):1148-1154

11. Linderoth B, Fedorcsak I, Meyerson BA (1991) Peripheral vasodilatation after spinal cord stimulation: animal studies of putative effector mechanisms. Neurosurgery 28(2):187-195

12. Mannheimer C, Carlsson CA, Emanuelsson H, Vedin A, Waagstein F, Wilhelmsson C (1985) The effects of transcutaneous electrical nerve stimulation in patients with severe angina pectoris. Circulation 71(2):308-316

13. Oakley JC, Prager JP (2002) Spinal cord stimulation: mechanisms of action. Spine 27(22):2574-2583

14. Padayachee TS, Kirkham FJ, Lewis RR, Gillard J, Hutchinson MCE, Gosling RG (1986) Transcranial measurement of blood velocities in the basal cerebral arteries using pulsed Doppler ultrasound: a method of assessing the Circle of Willis. Ultrasound Med Biol 12(1):5-14

15. Patel S (2004) Evidence for a central pathway in the cerebrovascular effects of spinal cord stimulation. Neurosurgery 55(1):201206

16. Pinheiro JC, Bates JM (2000) Mixed-effects models in S and SPLUS. Illustrated ed. Springer, New York

17. Sanderson JE, Tomlinson B, Lau MS, So KW, Cheung AH, Critchley JA, Woo KS (1995) The effect of transcutaneous electrical nerve stimulation (TENS) on autonomic cardiovascular reflexes. Clin Auton Res 5(2):81-84

18. Schwedt TJ, Matharu MS, Dodick DW (2006) Thunderclap headache. Lancet Neurol 5(7):621-631

19. Van Lieshout JJ, Secher NH (2008) Point:counterpoint: sympathetic activity does/does not influence cerebral blood flow. Point: sympathetic activity does influence cerebral blood flow. J Appl Physiol 105(4):1364-1366 
20. Van Lieshout JJ, Secher NH (2008) Last word on point: counterpoint: sympathetic activity does/does not influence cerebral blood flow. J Appl Physiol 105(4):1374

21. Vriens EM (1989) Transcranial pulsed Doppler measurements of blood velocity in the middle cerebral artery: reference values at rest and during hyperventilation in healthy volunteers in relation to age and sex. Ultrasound Med Biol 15(1):1-8

\section{Comment}

This study seeks to evaluate the efficacy and application of electromagnetic fields to modulate and treat cerebrovascular disease. The device of choice was the transcutaneous electrical neurostimulator (TENS); the end-point followed was cerebral blood flow in the middle cerebral artery, as measured by transcranial doppler ultrasonography. Within the 20 patients examined, no significant difference in outcomes was noted.

While simple, this paper provides a vital critique of popular mode of treatment. The use of electromagnetic and other noninvasive techniques to modify physiology has been proposed for over a century. More and more we see variations on this in products from industry designed to treat diseases without pills or surgery. The pursuit of non-invasive therapies is wonderful, but this article reminds us of the need to be rational and critical in our evaluation of novel therapies.

Markus Bookland, MD

Christopher M. Loftus MD

Philadelphia, USA 